\title{
VALIDATING A WORKFLOW FOR TREE INVENTORY UPDATING WITH 3D POINT CLOUDS OBTAINED BY MOBILE LASER SCANNING
}

\author{
Jinhu Wang $^{a, b, *}$, Roderik Lindenbergh ${ }^{a}$ \\ ${ }^{a}$ Dept. of Geoscience and Remote Sensing, Delft University of Technology \\ Building 23, Stevinweg 1, Post Box 5048, 2628CN Delft, The Netherlands \\ (jinhu.wang, r.c.lindenbergh)@ tudelft.nl \\ ${ }^{b}$ Regional Innovation Center Europe, Fugro. 2263 HW Leidschendam, The Netherlands
}

Commission II, WG II/10

KEY WORDS: Tree inventory, Mobile Laser Scanning, Point Cloud Data, Tree classification

\begin{abstract}
:
Urban trees are an important component of our environment and ecosystem. Trees are able to combat climate change, clean the air and cool the streets and city. Tree inventory and monitoring are of great interest for biomass estimation and change monitoring. Conventionally, parameters of trees are manually measured and documented in situ, which is not efficient regarding labour and costs. Light Detection And Ranging (LiDAR) has become a well-established surveying technique for the acquisition of geo-spatial information. Combined with automatic point cloud processing techniques, this in principle enables the efficient extraction of geometric tree parameters. In recent years, studies have investigated to what extend it is possible to perform tree inventories using laser scanning point clouds. Give the availability of a city of Delft Open data tree repository, we are now able to present, validate and extend a workflow to automatically obtain tree data from tree location until tree species. The results of a test over 47 trees show that the proposed methods in the workflow are able to individual urban trees. The tree species classification results based on the extracted tree parameters show that only one tree was wrongly classified using k-means clustering.
\end{abstract}

\section{INTRODUCTION}

Trees play an indispensable role in the recycling of material and energy between vegetation and the physical environment through photosynthesis and transpiration (Parker, 1995, Brunner, 1998). Tree inventory and management are of great interest for preserving ground biomass, monitoring environmental changes and assessing carbon sequestration for sustainable development (Cottone and Ettl, 2001, Zheng et al., 2007, Van Deusen, 2010, Moskal and Zheng, 2012, Baldocchi, 2013). Urban trees contribute to human health and well-beings in a variety of ways, such as removing pollutants and particulate matter (PM) from the air (Weber, 2013). Urban tree parameters, such as location, height, canopy projected area (CPA), diameter at breast height (DBH) and trunk length are important characteristics for tree management and monitoring in an increasingly urbanized world. However, manually measuring those parameters is inefficient regarding labour and cost.

Light Detection And Ranging (LiDAR) is able to quickly obtain 3D geometry and highly accurate point cloud data. A typical LiDAR system integrates a Global Navigation Satellite System (GNSS), an Inertial Measuring Unit (IMU) and laser scanning system. Point clouds acquired by LiDAR systems have been applied in a variety of applications. Examples are generating Digital Elevation Models (DEM) and Digital Terrain Models (DTM) (Vosselman, 2000, Pfeifer, 2001), 3D building reconstruction (Maas and Vosselman, 1999, Vosselman and Dijkman, 2001, Rutzinger et al., 2009, Xiong et al., 2014), land cover classification (Rutzinger et al., 2008, Guo et al., 2011, Mallet et al., 2011). Notably forest inventories and tree parameter estimation were performed based on point clouds obtained by ALS (Hyyppä et al., 2001, Persson et al., 2002, Gorte and Pfeifer, 2004, Morsdorf et al., 2006, Hu et al., 2017). However, due to the laser illuminating direction of ALS, point densities of the acquired point clouds are inadequate

${ }^{*}$ Corresponding author and tree trunks are not sampled well. It therefore hard to perform detailed tree parametrization.

However, a Mobile Laser Scanning (MLS) system has the aforementioned components to sample its surrounding environment and obtain highly accuracy and dense point clouds. Point clouds acquired by MLS have a variety of applications. A majority of the applications of MLS point cloud data focus on urban scenarios, such as road environment management (Pu et al., 2011, Puente et al., 2013, Cabo et al., 2014, Rodríguez-Cuenca et al., 2015, Wang et al., 2017) and urban roadside tree segmentation and classification (Jaakkola et al., 2010, Rutzinger et al., 2011, Puttonen et al., 2011, Sirmacek and Lindenbergh, 2015, Zhang et al., 2015, Weinmann et al., 2016).

In 2011, a low-cost system was developed and tested on tree measurements (Jaakkola et al., 2010). DBH and tree height were extracted but further classification and updating tree inventories was not conducted. In (Rutzinger et al., 2011), a method to construct tree models was proposed. Tree points were first classified in MLS point clouds and were further simplified. Then, model parameters such as stem diameter, lower crown diameter, middle crow diameter and upper crow diameter were extracted to model trees. However, tree species were not considered. In (Puttonen et al., 2011), a method for tree classification based on MLS point clouds and hyperspectral images was proposed. Experiments showed promising results on the classification, however, tree parameters were not extracted. An individual tree segmentation algorithm was presented in (Zhang et al., 2015). The method first segmented the raw MLS point cloud into ground and nonground points. Then, together with NDVI obtained from hyperspectral image, vegetation points were extracted. Next, tree points were obtained and the top of trees were extracted. Together with the crown diameter, the MLS points were labelled. Still, further tree parameters were not extracted and species were not considered. 
In this work, a workflow for tree inventory updating with 3D point clouds obtained by MLS is presented. The workflow first extract non-ground points from raw MLS point clouds and tree points are classified in the non-ground points. Consecutively, individual trees are delineated using a voxel-based method to acquire points of individual trees. Next, parameters of trees are estimated. Based on the extracted parameters, trees of different species are classified. The final step is to validate the obtained results and update an existing tree database.

\section{METHODOLOGY}

The methodology described in this works consists of five steps. (1) segment non-ground points from raw MLS point clouds. (2) classify tree points. (3) individual tree delineation. (4) tree parameter estimation. (5) parameter normalization and (6) validate results and update the existing database. The steps are described in detail in the following sections.

\subsection{Segment non-ground points}

The first step is ground and non-ground points separation. This procedure is also known as filtering of point clouds. In this work, the separation of non-ground points was conducted by using an existing algorithm (Kraus and Pfeifer, 1998). The algorithm is originally designed for filtering point clouds obtained by airborne laser scanners. It iteratively computes a weight function and by a pre-set threshold, the terrain is obtained and ground and nonground points are separated. Due to the huge number and high density of points obtained by mobile laser scanners, it is considered to design a computationally more efficient alternative.

\subsection{Classify tree points}

Tree point classification in this work is conducted by the algorithm presented in (Sirmacek and Lindenbergh, 2015). The algorithm takes the non-ground points as input and projects all nonground points onto a horizontal plane. Then, by estimating the 2D density of the projected points, local maxima are acquired that correspond to the locations of trees. Afterwards, tree points are assigned to each tree in the neighbourhood. Although some façade points are wrongly classified as tree points, the algorithm works well for point clouds that are re-tiled with regard to a distance across and along the scanning trajectory.

\subsection{Individual tree delineation}

Individual tree delineation is performed using a voxel based algorithm presented in (Wang, 2017). The algorithm takes the tree points obtained from the previous step as input. Then, the tree points are re-sampled using voxels with pre-set voxel sizes in the three coordinate axes. Next, seed voxels are identified by the second input parameter, minimum tree diameter. The algorithm traverses the voxels either from the top voxel layer downwards or from the bottom layer upwards to assign voxels to the designated voxel clusters. Finally, tree points are obtained from the voxel clusters. Notably, the traversing direction in this work is from the bottom layer upwards since the trunk of trees near the scanning trajectory are well sampled.

\subsection{Tree parameter estimation}

In this work, tree parameters are estimated using a novel voxel based method. In this work, the estimated parameters for each tree consist of the location of trees, tree height, trunk length and canopy projected area on the ground, as illustrated in Figure 1.

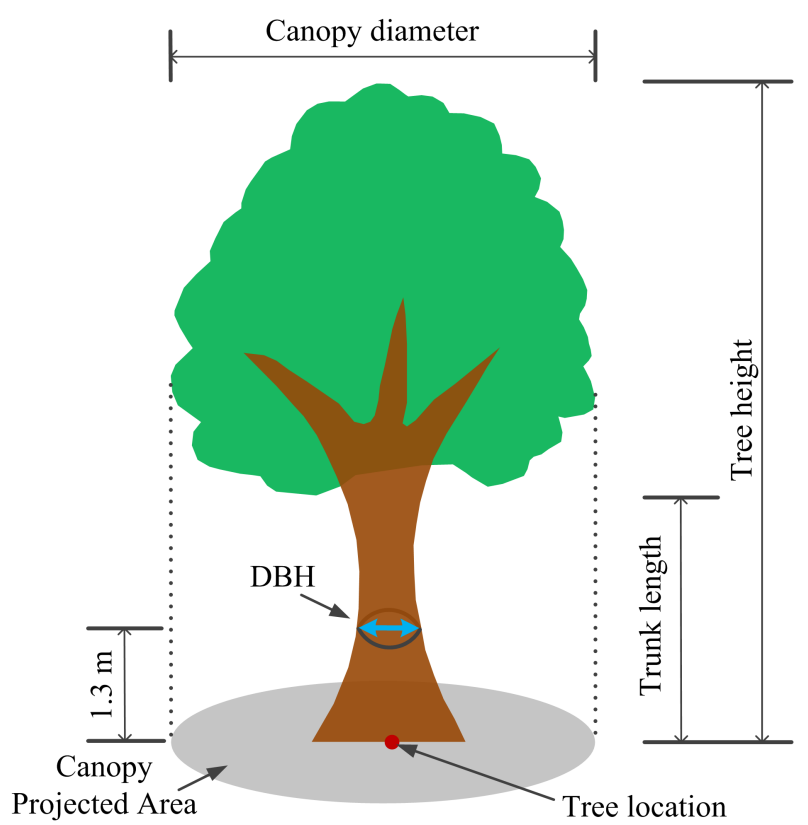

Figure 1: An illustration of tree parameters extracted in this study.

Location and height are estimated by determining the position of the points at the bottom of each trunk and the vertical extend of the tree points respectively. Notably, the length of trunks was estimated by determining the maximum gradient of the number of voxels at each vertical layer. Figure 2 shows an example of a voxelization of one tree. Figure $2(a)$ and $(b)$ are projections on plane $X O Z$ and $Y O Z$ respectively. There are 30 vertical layers and tree points are colored from blue to red by height.

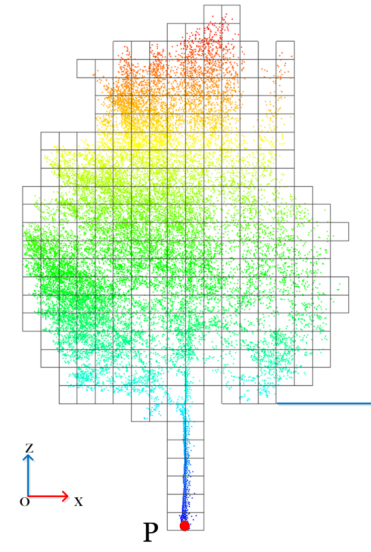

(a)

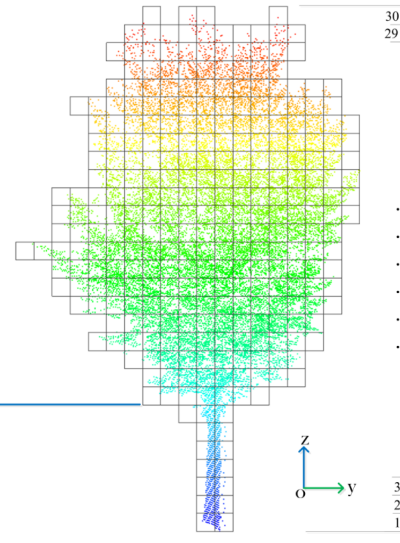

(b)
Figure 2: Strategy to estimate the location and trunk length of a tree.

The tree trunk starts at the bottom layer. First the voxel cells are projected on plane $x o z$ and yoz, as shown in Figure $2(a)$ and $(b)$. Then the number of cells in each layer is calculated as $f(i)$ and $g(i)$ respectively, where $i=1,2, \ldots, n$ denotes the layer index. In this study, the trunk length ends at the $k-t h$ layer, in which there is a dramatic increase in the number of voxel cells. $k$ is determined by Formula 1, where $h(i)=f(i)+g(i)$ refers to the total total number of cells in the two projected planes in each layer. Then the trunk length is determined by $L_{\text {trunk }}=$ $k \times Z_{\text {size }}$. 


$$
k=\underset{i}{\arg \max } h^{\prime}(i)=\underset{i}{\arg \max }\{f(i)+g(i)\}^{\prime}
$$

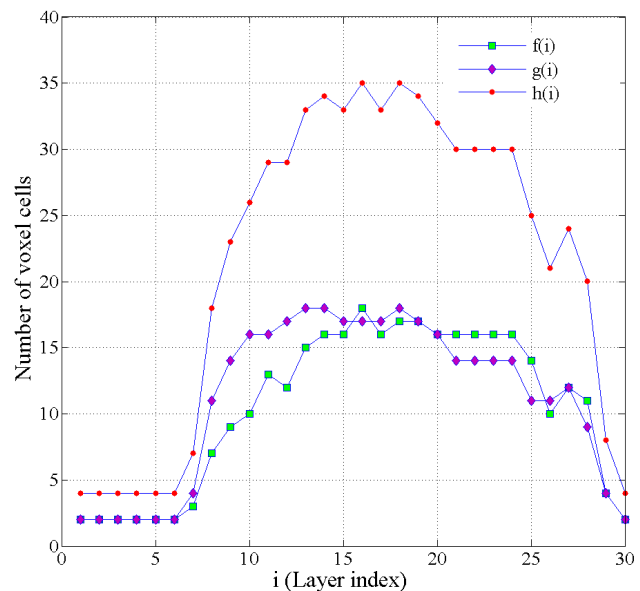

Figure 3: Number of cells in each layer.

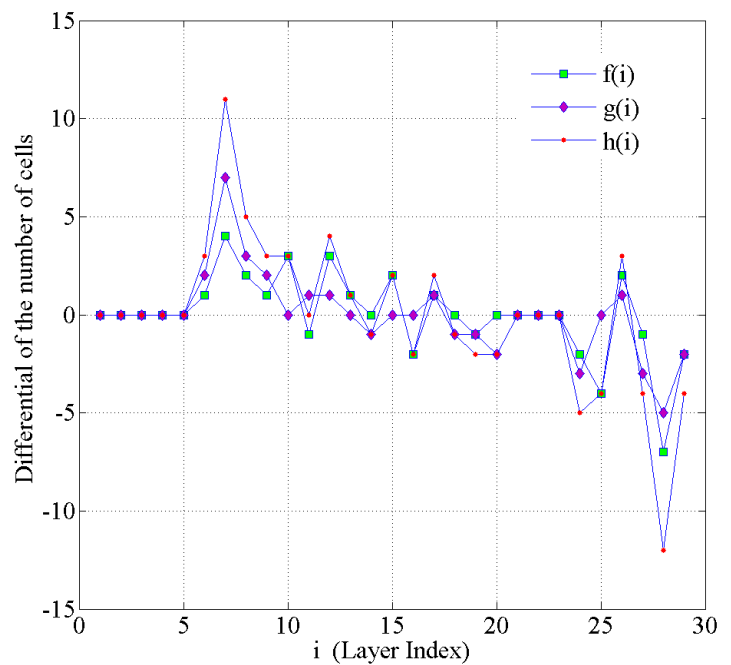

Figure 4: Fist degree differential of the number of cells in each layer.

Figure 3 shows the number of cells in each layer for the scenario shown in Figure 2. The number of cells in each layer of the scenario illustrated in Figure 2 is plotted in Figure 3, where $f(i)$, $g(i)$ and $h(i)$ are the same as in Equation 1. Figure 4 is the illustration of the differential of $f(i), g(i)$ and $h(i)$, the layer which has maximum value is the the highest point of the tree trunk. In this specific scenario, the $7-t h$ layer is estimated as the top of the trunk of this tree.

The canopy projection area is estimated by first projecting points onto a horizontal plane. Then, the boundary of the points is estimated by alpha shape (Edelsdrunner and Kirkpatric, 1983) with an alpha value of 0.8 . Although the further part of tree canopy is not well sample due to occlusions, the point density is enough to represent the shape of a tree canopy. The DBH is the diameter of the trunk at a height of 1.3 meter above ground. In this work, trunk points that have a local height between $1.2 \mathrm{~m}$ and $1.4 \mathrm{~m}$ are used and projected onto horizontal plane. Then a circle fitting step is performed to obtain the diameter of the best fit circle. As shown in Figure 5, the blue points are the tree trunk points and the red circle is the best fit circle. The green dot is the estimated centre of the circle.

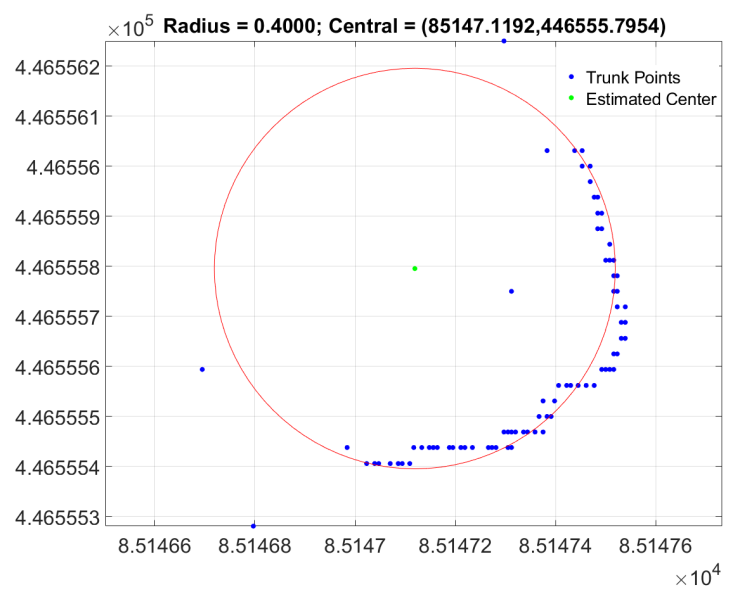

Figure 5: Estimating the DBH of a tree.

\subsection{Normalized tree parameters}

The tree parameters extracted in Section 2.4 are used to classify the species of trees. To avoid the influence of the size of a tree on a specific parameter, the estimated parameters are normalized. That is, the normalized CPA, i.e. $C P A_{N}$, is calculated by dividing the extracted CPA with the estimated tree height, as given in Equation 2. The normalized trunk length Length $_{N}$ is determined by dividing the estimated trunk length with the tree height. The same procedure are applied to the obtained DBH.

$$
\begin{aligned}
C P A_{N} & =\frac{C P A}{\text { Height }} \\
\text { Length }_{N} & =\frac{\text { Length }}{\text { Height }} \\
D B H_{N} & =\frac{\text { DBH }}{\text { Height }}
\end{aligned}
$$

\subsection{Validate results and update the existing database}

The final step of this work is to validate the obtained results and update the existing tree database. To validate the results, trees along an urban roadside around TU Delft campus were selected and scanned by a MLS system, the Fugro Drive-Map system. There is also an open database of the trees that are owned by the Delft municipality and are therefore documented. Other trees belong to TU Delft and thus were not included yet in this database. Validation of the extracted tree parameters will be conducted for the trees in the cadastre database. The results will also be used to update the database.

\section{RESULTS AND DISCUSSION}

\subsection{Data description}

The point cloud data analysed in this work is acquired by the Fugro Drive-Map MLS system. In this work, three species of trees were considered. Figure 6 shows the 47 studied trees. The number of trees corresponding to Figure $6(a),(b)$ and $(c)$ are 14, 15 
and 18 and the species of the trees are Aesculus hippocastanum 'Baumannii', Acer saccharinum and Platanus x hispanica respectively.
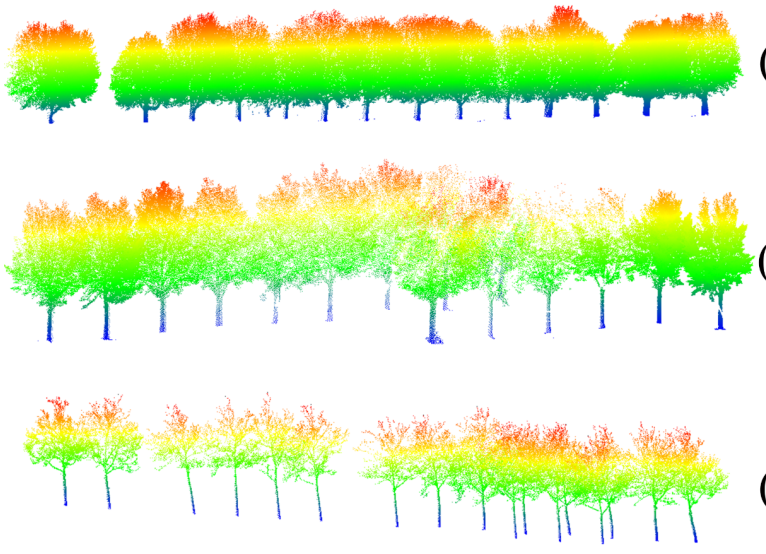

Figure 6: Three species of trees are studied in this work.

\subsection{Results of tree parameter estimation}

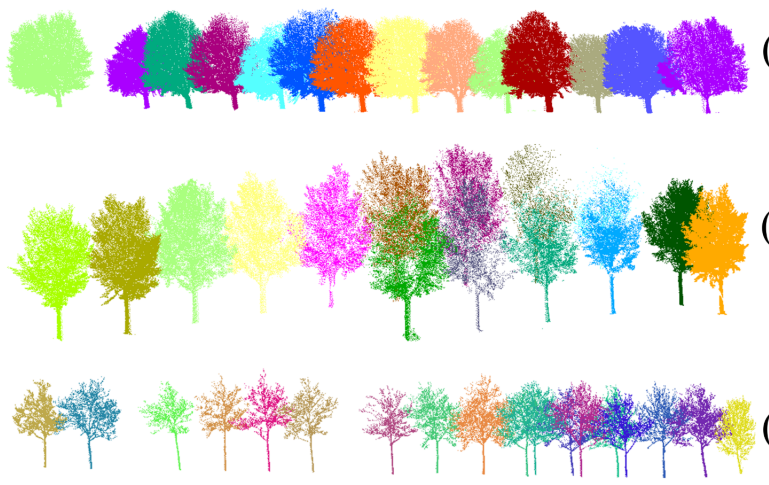

Figure 7: Individual tree delineation results of the studied 47 trees.

The estimation of tree parameters starts with the separation of individual trees. In this work, the voxel size was set to $50 \mathrm{~cm}$ in the three coordinate directions. The minimum tree diameter was set to $8.5 \mathrm{~m}, 3.5 \mathrm{~m}$ and $2.5 \mathrm{~m}$ corresponding to the trees in Figure 6 $(a),(b)$ and $(c)$ respectively. Figure 7 shows the individual tree delineation results.

Table 1, Table 2 and Table 3 are the estimated tree parameters. Note that normalized CPA and normalized DBH are the extracted $\mathrm{CPA}$ and $\mathrm{DBH}$ divided by the tree height.

The results in Table 1 to Table ?? show that for example the Aesculus hippocastanum 'Baumannii' is quite compact tree. This is reflected by a bigger normalized DBH and a bigger normalized CPA. The trees of the other species are more alike, but still have distinguishable normalized tree parameters.

The next step is to perform automatic tree classification based on the extracted tree parameters. In this work, k-means clustering is employed to classify the trees. The number of classes is set to 3 and the implementation in MATLAB is employed. The classification results are shown in Figure 8.

In the figure, the circles are the ground truth of tree species and the colors of the triangles are the automatically clustered trees.
Table 1: Tree parameter estimation results from the trees of species Aesculus hippocastanum 'Baumannii' in Figure $7 a$

\begin{tabular}{ccccccc}
\hline Tree ID & Height & Trunk length & CPA & DBH & Norm. CPA & Norm. DBH \\
\hline 0 & 15.94 & 2.27 & 162.65 & 0.74 & 10.21 & 0.047 \\
1 & 14.06 & 1.92 & 119.99 & 0.63 & 8.54 & 0.045 \\
2 & 16.67 & 2.31 & 130.98 & 0.81 & 7.86 & 0.048 \\
3 & 16.37 & 2.96 & 129.11 & 0.70 & 7.89 & 0.043 \\
4 & 14.60 & 2.08 & 133.45 & 0.79 & 9.14 & 0.054 \\
5 & 17.28 & 2.47 & 149.74 & 1.04 & 8.67 & 0.060 \\
6 & 16.18 & 2.80 & 157.83 & 0.97 & 9.75 & 0.060 \\
7 & 16.41 & 2.82 & 157.35 & 0.96 & 9.59 & 0.059 \\
8 & 15.52 & 2.67 & 113.79 & 0.80 & 7.33 & 0.052 \\
9 & 14.74 & 2.62 & 128.34 & 0.62 & 8.71 & 0.042 \\
10 & 17.19 & 2.55 & 173.50 & 0.87 & 10.10 & 0.051 \\
11 & 13.40 & 2.62 & 70.04 & 0.66 & 5.23 & 0.049 \\
12 & 15.60 & 2.90 & 123.46 & 0.80 & 7.91 & 0.051 \\
13 & 16.03 & 2.67 & 149.03 & 0.98 & 9.30 & 0.061 \\
\hline Mean & 15.71 & 2.55 & 135.67 & 0.81 & 8.59 & 0.0515 \\
std.dev. & 1.15 & 0.13 & 26.02 & 0.14 & 1.31 & 0.0065 \\
\hline
\end{tabular}

Table 2: Tree parameter estimation results from the trees of species Acer saccharinum in Figure $7(b)$

\begin{tabular}{ccccccc}
\hline Tree ID & Height & Trunk length & CPA & DBH & Norm. CPA & Norm. DBH \\
\hline 0 & 14.27 & 3.34 & 57.62 & 0.39 & 4.04 & 0.027 \\
1 & 14.71 & 3.36 & 52.78 & 0.45 & 3.59 & 0.030 \\
2 & 15.03 & 3.50 & 70.84 & 0.48 & 4.71 & 0.032 \\
3 & 14.87 & 3.26 & 60.01 & 0.37 & 4.04 & 0.025 \\
4 & 14.71 & 3.42 & 70.62 & 0.57 & 4.80 & 0.039 \\
5 & 15.87 & 3.75 & 63.16 & 0.43 & 3.98 & 0.027 \\
6 & 15.04 & 3.32 & 63.78 & 0.47 & 4.24 & 0.031 \\
7 & 14.60 & 4.11 & 49.58 & 0.37 & 3.40 & 0.025 \\
8 & 12.37 & 2.88 & 48.53 & 0.38 & 3.92 & 0.030 \\
9 & 13.66 & 3.43 & 45.71 & 0.35 & 3.35 & 0.025 \\
10 & 13.46 & 4.02 & 50.53 & 0.34 & 3.76 & 0.025 \\
11 & 14.08 & 4.49 & 41.26 & 0.31 & 2.93 & 0.022 \\
12 & 15.21 & 3.94 & 56.45 & 0.34 & 3.71 & 0.022 \\
13 & 16.41 & 3.98 & 58.25 & 0.35 & 3.55 & 0.021 \\
14 & 15.91 & 4.16 & 67.14 & 0.43 & 4.22 & 0.027 \\
\hline Mean & 14.68 & 3.67 & 57.08 & 0.40 & 3.88 & 0.027 \\
std.dev. & 1.03 & 0.44 & 9.00 & 0.07 & 0.50 & 0.0046
\end{tabular}

Table 3: Tree parameter estimation results from the trees of species Platanus $x$ hispanica in Figure $7(c)$

\begin{tabular}{ccccccc}
\hline Tree ID & Height & Trunk Length & CPA & DBH & Norm. CPA & Norm. DBH \\
\hline 0 & 10.32 & 3.23 & 38.90 & 0.27 & 3.77 & 0.026 \\
1 & 9.68 & 3.28 & 26.47 & 0.20 & 2.74 & 0.020 \\
2 & 11.03 & 3.22 & 42.27 & 0.21 & 3.83 & 0.019 \\
3 & 10.12 & 3.11 & 35.83 & 0.22 & 3.54 & 0.022 \\
4 & 10.65 & 3.49 & 35.54 & 0.20 & 3.34 & 0.019 \\
5 & 10.13 & 3.13 & 31.41 & 0.19 & 3.10 & 0.019 \\
6 & 10.40 & 3.32 & 43.44 & 0.23 & 4.18 & 0.022 \\
7 & 10.06 & 3.31 & 36.06 & 0.23 & 3.59 & 0.023 \\
8 & 9.83 & 2.76 & 38.03 & 0.20 & 3.87 & 0.020 \\
9 & 8.62 & 2.22 & 15.20 & 0.13 & 1.76 & 0.015 \\
10 & 10.58 & 3.09 & 37.00 & 0.20 & 3.50 & 0.019 \\
11 & 10.06 & 3.00 & 31.40 & 0.19 & 3.12 & 0.019 \\
12 & 10.38 & 3.26 & 34.12 & 0.21 & 3.29 & 0.020 \\
13 & 9.68 & 2.61 & 30.25 & 0.20 & 3.12 & 0.020 \\
14 & 10.68 & 4.20 & 28.92 & 0.19 & 2.71 & 0.018 \\
15 & 11.34 & 3.52 & 28.16 & 0.17 & 2.48 & 0.015 \\
16 & 9.76 & 3.27 & 21.15 & 0.19 & 2.17 & 0.019 \\
17 & 9.78 & 3.94 & 26.37 & 0.21 & 2.70 & 0.021 \\
\hline Mean & 10.17 & 3.22 & 32.25 & 0.20 & 3.15 & 0.0198 \\
std.dev. & 0.60 & 0.44 & 7.00 & 0.03 & 0.63 & 0.0026 \\
\hline
\end{tabular}

By comparing to the ground truth, there is only one tree of type Aesculus hippocastanum 'Baumannii' that was wrongly classified to tree type Acer saccharinum, which is noted in Figure 8 by the green rectangle.

\section{CONCLUSIONS}

In this work, a workflow for tree parameter estimation and tree cadastre database updating is presented and validated based on point clouds obtained by mobile laser scanner. The validation was conducted with respect to an existing open tree cadastre database. Then, updating of the tree cadastre database was performed based on the extracted results. A possible further application scenario is to automatically identify the species of trees based on the extracted tree parameters, as all the trees in the cadastre database also have species information. 


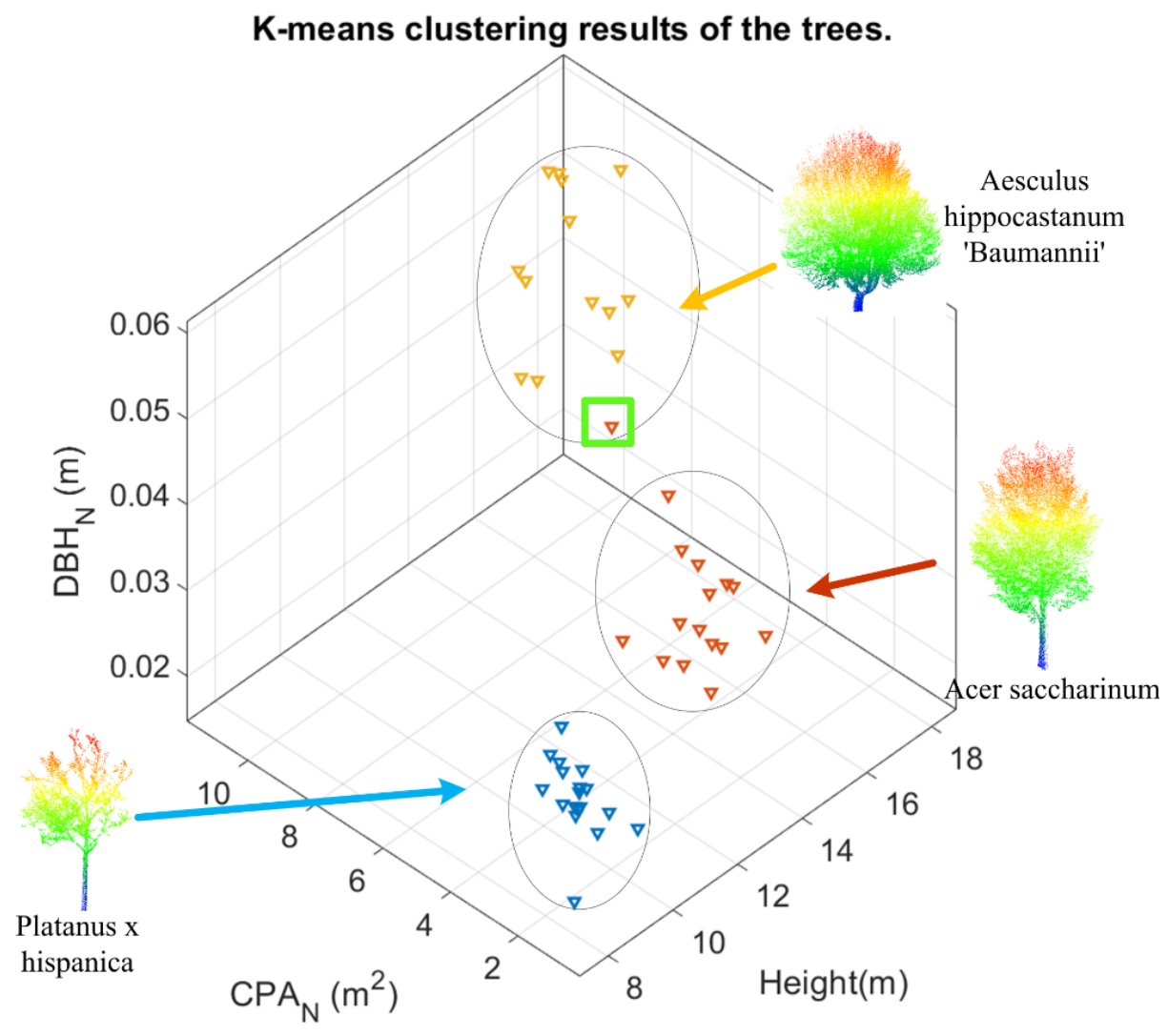

Figure 8: Automatic k-means unsupervised classification of trees, using normalized tree parameters.

\section{REFERENCES}

Baldocchi, D., 2013. 4.08 - ecosystem services of energy exchange and regulation. In: R. A. Pielke (ed.), Climate Vulnerability, Academic Press, Oxford, pp. $81-92$.

Brunner, A., 1998. A light model for spatially explicit forest stand models.

Cabo, C., Ordoñez, C., García-Cortés, S. and Martínez, J., 2014. An algorithm for automatic detection of pole-like street furniture objects from Mobile Laser Scanner point clouds. ISPRS Journal of Photogrammetry and Remote Sensing 87, pp. 47-56.

Cottone, N. and Ettl, G. J., 2001. Estimating populations of whitebark pine in Mount Rainier National Park, Washington, using aerial photography. Northwest Science 75(4), pp. 397-406.

Edelsdrunner, H. and Kirkpatric, D. G., 1983. On the sahpe of a set of points in the plane. IEEE Transactions On Inforamtion Theory 29(4), pp. 551-559.

Gorte, B. and Pfeifer, N., 2004. Structuring laser-scanned trees using 3D mathematical morphology. International Archives of Photogrammetry and Remote Sensing 35, pp. 929-933.

Guo, L., Chehata, N., Mallet, C. and Boukir, S., 2011. Relevance of airborne lidar and multispectral image data for urban scene classification using Random Forests. ISPRS Journal of Photogrammetry and Remote Sensing 66(1), pp. 56-66.

Hu, S., Li, Z., Zhang, Z., He, D. and Wimmer, M., 2017. Efficient tree modeling from airborne LiDAR point clouds. Computers and Graphics (Pergamon) 67, pp. 1-13.
Hyyppä, J., Kelle, O., Lehikoinen, M. and Inkinen, M., 2001 A segmentation-based method to retrieve stem volume estimates from 3-D tree height models produced by laser scanners. IEEE Transactions on Geoscience and Remote Sensing 39(5), pp. 969975.

Jaakkola, A., Hyyppä, J., Kukko, A., Yu, X., Kaartinen, H., Lehtomäki, M. and Lin, Y., 2010. A low-cost multi-sensoral mobile mapping system and its feasibility for tree measurements. ISPRS Journal of Photogrammetry and Remote Sensing 65(6), pp. 514-522.

Kraus, K. and Pfeifer, N., 1998. Determination of terrain models in wooded areas with airborne laser scanner data. ISPRS Journal of Photogrammetry and Remote Sensing 53(4), pp. 193-203.

Maas, H. G. and Vosselman, G., 1999. Two algorithms for extracting building models from raw laser altimetry data. ISPRS Journal of Photogrammetry and Remote Sensing 54(2-3), pp. 153-163.

Mallet, C., Bretar, F., Roux, M., Soergel, U. and Heipke, C., 2011. Relevance assessment of full-waveform lidar data for urban area classification. ISPRS Journal of Photogrammetry and Remote Sensing.

Morsdorf, F., Kötz, B., Meier, E., Itten, K. I. and Allgöwer, B., 2006. Estimation of LAI and fractional cover from small footprint airborne laser scanning data based on gap fraction. Remote Sensing of Environment 104(1), pp. 50-61.

Moskal, L. M. and Zheng, G., 2012. Retrieving forest inventory variables with terrestrial laser scanning (TLS) in urban heterogeneous forest. Remote Sensing 4(1), pp. 1-20. 
Parker, G. G., 1995. Structure and microclimate of forest canopies. In: M. Lowman and N. Nadkami (eds), Forest canopies: a review of research on a biological frontier, Academic Press.

Persson, A., Holmgren, J. and Söderman, U., 2002. Detecting and measuring individual trees using an airborne laser scanner. Photogrammetric Engineering \& Remote Sensing 68(9), pp. 925932

Pfeifer, N., 2001. Derivation Of Digital Terrain Models In The Scop++ Environment. OEEPE Workshop on Airborne Laserscanning and Interferometric SAR for Digital Elevation Models, , p. 13.

$\mathrm{Pu}$, S., Rutzinger, M., Vosselman, G. and Oude Elberink, S., 2011. Recognizing basic structures from mobile laser scanning data for road inventory studies. ISPRS Journal of Photogrammetry and Remote Sensing.

Puente, I., González-Jorge, H., Martínez-Sánchez, J. and Arias, P., 2013. Review of mobile mapping and surveying technologies. Measurement 46(7), pp. 2127-2145.

Puttonen, E., Jaakkola, A., Litkey, P. and Hyyppä, J., 2011. Tree classification with fused mobile laser scanning and hyperspectral data. Sensors 11(5), pp. 5158-5182.

Rodríguez-Cuenca, B., García-Cortés, S., Ordóñez, C. and Alonso, M., 2015. Automatic Detection and Classification of Pole-Like Objects in Urban Point Cloud Data Using an Anomaly DetectionAlgorithm. Remote Sensing 7(10), pp. 12680-12703.

Rutzinger, M., Höfle, B., Hollaus, M. and Pfeifer, N., 2008. Object-based point cloud analysis of full-waveform airborne laser scanning data for urban vegetation classification. Sensors 8(8), pp. $4505-4528$.

Rutzinger, M., Pratihast, A. K., Oude Elberink, S. J. and Vosselman, G., 2011. Tree modelling from mobile laser scanning data-sets. Photogrammetric Record 26(135), pp. 361-372.

Rutzinger, M., Rottensteiner, F. and Pfeifer, N., 2009. A Comparison of Evaluation Techniques for Building Extraction From Airborne Laser Scanning. IEEE Journal of Selected Topics in Applied Earth Observations and Remote Sensing 2(1), pp. 1120

Sirmacek, B. and Lindenbergh, R., 2015. Automatic classification of trees from laser scanning point clouds. ISPRS Annals of Photogrammetry, Remote Sensing and Spatial Information Sciences II-3/W5, pp. 137-144.

Van Deusen, P., 2010. Carbon sequestration potential of forest land: Management for products and bioenergy versus preservation. Biomass and Bioenergy 34(12), pp. 1689-1694.

Vosselman, G., 2000. Slope based filtering of laser altimetry data. In: IAPRS 2000 , pp. 1-8.

Vosselman, G. and Dijkman, S., 2001. 3D building model reconstruction from point clouds and ground plans. Int. Arch. of Photogrammetry and Remote Sensing XXXIV, pp. 37-43.

Wang, J., Lindenbergh, R. and Menenti, M., 2017. Sigvox - a $3 \mathrm{~d}$ feature matching algorithm for automatic street object recognition in mobile laser scanning point clouds. ISPRS Journal of Photogrammetry and Remote Sensing 128, pp. $111-129$.

Weber, C., 2013. Ecosystem services provided by urban vegetation: A literature review. In: S. Rauch, G. Morrison, S. Norra and N. Schleicher (eds), Urban Environment, Springer Netherlands, Dordrecht, pp. 119-131.
Weinmann, M., Mallet, C. and Bredif, M., 2016. Detection, segmentation and localization of individual trees from mms point cloud data.

Xiong, B., Oude Elberink, S. and Vosselman, G., 2014. A graph edit dictionary for correcting errors in roof topology graphs reconstructed from point clouds. ISPRS Journal of Photogrammetry and Remote Sensing 93, pp. 227-242.

Zhang, C., Zhou, Y. and Qiu, F., 2015. Individual Tree Segmentation from LiDAR Point Clouds for Urban Forest Inventory. Remote Sensing 7(6), pp. 7892-7913.

Zheng, G., Chen, J. M., Tian, Q. J., Ju, W. M. and Xia, X. Q., 2007. Combining remote sensing imagery and forest age inventory for biomass mapping. Journal of Environmental Management 85(3), pp. 616-623. 